\title{
Endothelial cell Pannexin1 modulates severity of ischemic stroke by regulating cerebral inflammation and myogenic tone
}

Miranda E Good, ${ }^{1}$ Stephanie A. Eucker, ${ }^{2}$ Jun Li, ${ }^{3}$ Hannah M. Bacon, ${ }^{1}$ Susan M. Lang, ${ }^{1}$ Joshua T. Butcher, Tyler J. Johnson, ${ }^{1}$ Ronald P. Gaykema, ${ }^{3}$ Manoj K. Patel, ${ }^{3}$ Zhiyi Zuo, ${ }^{3}$ and Brant E. Isakson ${ }^{1,4}$

'Robert M. Berne Cardiovascular Research Center, University of Virginia School of Medicine, Charlottesville, Virginia, USA 2Division of Emergency Medicine, Department of Surgery, Duke University, Durham, North Carolina, USA. ${ }^{3}$ Department of Anesthesiology and ${ }^{4}$ Department of Molecular Physiology and Biophysics, University of Virginia School of Medicine, University of Virginia School of Medicine, Charlottesville, Virginia, USA.

Ischemic stroke is a leading cause of morbidity and mortality in the US; however, there currently exists only one effective acute pharmacological therapeutic intervention. Purinergic signaling has been shown to regulate vascular function and pathological processes, including inflammation and arterial myogenic reactivity, and plays a role in ischemic stroke outcome. Purinergic signaling requires extracellular purines; however, the mechanism of purine release from cells is unclear. Pannexin1 (Panx1) channels are potentially novel purine release channels expressed throughout the vascular tree that couples regulated purine release with purinergic signaling. Therefore, we examined the role of smooth muscle and endothelial cell Panx1, using conditional cell type-specific transgenic mice, in cerebral ischemia/reperfusion injury outcomes. Deletion of endothelial cell Panx1, but not smooth muscle cell Panx1, significantly reduced cerebral infarct volume after ischemia/reperfusion. Infiltration of leukocytes into brain tissue and development of cerebral myogenic tone were both significantly reduced when mice lacked endothelial Panx1. Panx1 regulation of myogenic tone was unique to the cerebral circulation, as mesenteric myogenic reactivity and blood pressure were independent of endothelial Panx1. Overall, deletion of endothelial Panx1 mitigated cerebral ischemic injury by reducing inflammation and myogenic tone development, indicating that endothelial Panx 1 is a possible novel target for therapeutic intervention of ischemic stroke.

Conflict of interest: The authors have declared that no conflict of interest exists.

Submitted: July 14, 2017 Accepted: February 16, 2018 Published: March 22, 2018

Reference information: JCI Insight. 2018;3(6):e96272. https:// doi.org/10.1172/jci.insight.96272.

\section{Introduction}

Stroke is the fourth leading cause of death in the US, and each year, approximately 15 million people experience an acute ischemic stroke worldwide (1). Although the incidence of acute ischemic stroke is remarkably high, acute pharmacological treatment remains limited to thrombolytic therapy. Thrombolytic agents such as tissue plasminogen activator (tPA) break down vascular obstructions but do not aim to reduce the extent of the injury following reperfusion. Additionally, the short window of effectiveness for administration of tPA drastically limits its therapeutic potential (2). Identification of novel therapeutic targets is necessary to improve patient outcomes following an ischemic stroke. Recently, pannexins have emerged as potentially novel channels within multiple cell types of the brain that might provide such a target for improving stroke outcome.

Pannexins are a recently discovered family of membrane channels whose primary function appears to be the regulated release of purines from intracellular stores (3). There are 3 known pannexin isoforms, Pannexin1 (Panx1), Panx2, and Panx3. The Panx1 isoform is found in endothelial cells (ECs) throughout the arterial tree, as well as in smooth muscle cells (SMCs) of arteries and arterioles less than $300 \mu \mathrm{m}$ in diameter (4). Although Panx1 continues to be discovered in new locations, a complete understanding of the role(s) these channels play in regulating vascular function is currently lacking.

Panx1 is present in both the neurons and the cerebral vasculature of the rat (5). Deletion or blocking of pannexins has been shown to significantly improve outcome following middle cerebral artery occlusion (MCAO) (6-8); nevertheless, these studies have important limitations. Systemically administered 
pharmacological inhibitors of pannexins significantly reduced infarct size following $\mathrm{MCAO} /$ reperfusion $(6,8)$. In addition, global deletion of Panx1 and Panx2 reduced infarct volume following permanent MCAO (7). However, global deletion of Panx1 was also shown to upregulate Panx3 in the vasculature, indicating a potential compensatory mechanism (9). Furthermore, the focus of these studies was on the pannexins found in neurons and the effects of ATP on neuronal function. While these data point to pannexins playing a significant role in determining stroke outcome, the studies globally affected pannexin channels within the brain, including those in the vascular cells, indicating the need to explore the role of Panx1 specifically in the cerebral vasculature.

Purine release by Panx1 channels has been shown to regulate vascular function in both venous and arterial vessels $(10,11)$. For instance, endothelial Panx1 regulates TNF- $\alpha$-induced leukocyte adhesion and emigration in venules (11). TNF- $\alpha$ is released following an ischemic stroke, and treatment with an antiTNF- $\alpha$ antibody or inhibition of neutrophil infiltration improves stroke outcome in mice, indicating a role of inflammation in determining severity of stroke $(12,13)$.

In addition to the venous inflammatory pathway, upstream regulation of cerebral blood flow (CBF) by Panx1 may also impact stroke outcome. Restoring adequate CBF is vital for recovery following acute ischemic stroke (14-16). CBF is largely regulated by myogenic reactivity, in which arteries constrict in response to increased luminal pressure. Myogenic reactivity is altered in pathophysiological states such as cerebral ischemia (17), so understanding its mechanisms is crucial. Recent reports suggest that myogenic reactivity is a purinergic-dependent mechanism, specifically through P2Y receptors that are activated by extracellular purines (e.g., ATP) $(14,18,19)$. Therefore, mechanisms that couple the release of ATP with activation of $\mathrm{P} 2 \mathrm{Y}$ receptors may be targets for pharmacological interventions to improve $\mathrm{CBF}$ regulation in disease states.

In this study, we reveal a potentially novel mechanism by which vascular Panx1 regulates stroke outcome in mice. We demonstrate that deletion of endothelial, but not smooth muscle, Panx1 significantly improves stroke outcome following cerebral ischemia/reperfusion injury by reducing infiltration of circulating leukocytes and reducing the development of myogenic tone in cerebral arteries. Furthermore, by showing the absence of Panx1 effects on peripheral mesenteric artery myogenic tone development, we demonstrate that endothelial Panx1 regulation of myogenic tone is unique to the cerebral arteries.

\section{Results}

Endothelial Panx1 protects against cerebral ischemia/reperfusion injury. Panx1 is expressed in multiple cell types within the brain $(5,20,21)$. Here, we demonstrate Panx1 staining throughout the brain (Figure 1A), and we find high expression within the neurons (Figure 1B). Posterior cerebral arteries (PCAs) isolated from control mice had Panx1 expression in EC and SMC (Figure 1, C and F). Secondary and isotype controls were negative for staining (Figure 1, D and E). Mice with activated Cre specific to EC or SMC have reduced staining in their respective cell types (Figure 1, G and $\mathrm{H}$ ). To determine the potential role of vascular Panx1 in regulating vascular response to ischemic stroke, we performed a 90-minute unilateral MCAO followed by 24-hour reperfusion in transgenic mice lacking Panx1 in either SMCs (SMC Panx1 $\Delta / \Delta ; 1$ Figure 2, B and C) or ECs (EC Panx1 $\Delta / \Delta$; Figure 2, E and G), and their controls (SMC Panx1 fl/fl or EC Panx1 fl/fl, respectively, and $\mathrm{Cre}^{-} \mathrm{Panx} 1 \mathrm{fl} / \mathrm{fl}$ [tamoxifen control]; Figure 2, A, D, and F) and stained with 2,3,5-triphenyltetrazolium chloride (TTC) to quantify infarct volume (Figure 2, A-F). Deletion of SMC Panx1 did not change the infarct volume following the ischemic stroke; however, deletion of EC Panx1 significantly mitigated the infarct volume (Figure 2, C and G).

Infiltration of circulating leukocytes is significantly reduced in mice lacking endothelial Panx1. EC Panx1 has previously been shown to regulate TNF- $\alpha$-mediated leukocyte rolling and adhesion, and inflammation is known to occur following an ischemia/reperfusion insult in the brain (11, 22-24). Thus, we examined the infiltration of circulating leukocytes into ischemic brain tissue of Panx1 transgenic mice using flow cytometry (Figure 3, D and E). EC Panx1 fl/fl mice showed an increased infiltration of leukocytes, which express high levels of CD45 (CD45 hi ), in the ischemic hemisphere compared with the contralateral hemisphere (Figure 3A). Of these infiltrating leukocytes, $\mathrm{Ly}_{6 \mathrm{G}}{ }^{+}$cells (neutrophils) were the predominant cell type (Figure 3B), whereas CD11b and Ly6C double-positive cells (CD11 b ${ }^{+} / \mathrm{Ly}_{6} \mathrm{C}^{+}$; monocytes) were minimally present (Figure 3C). Deletion of EC Panx1 significantly reduced the total percentage of infiltrating leukocytes but did not significantly change the relative amounts of the 2 specific types of leukocytes examined. Reduction of MCA blood flow in these leukocyte infiltration studies was verified using laser 


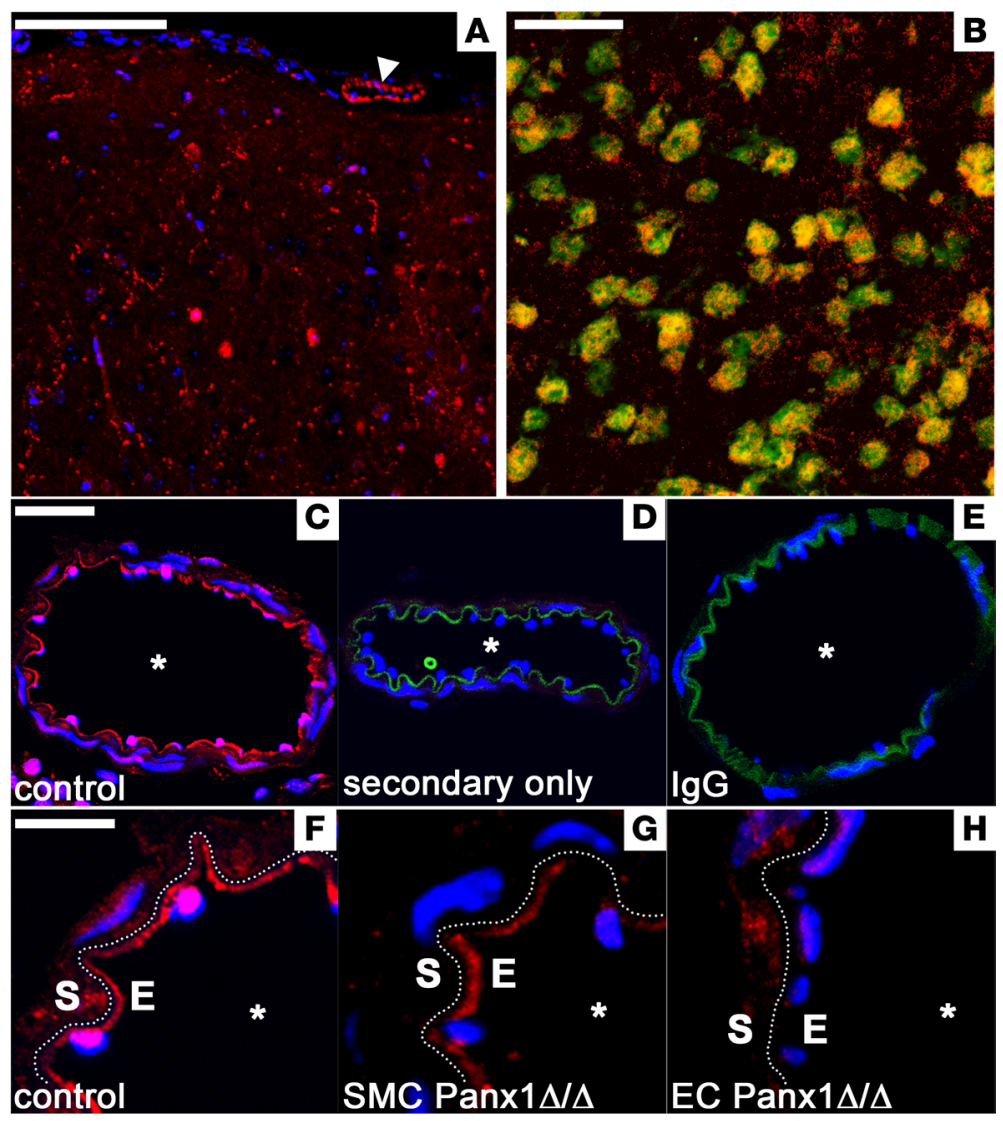

Figure 1. Panx1 is expressed in EC and SMC of cerebral arteries with successful knockdown in EC and SMC transgenic mice. In all sections, Panx1 (Panx1-CT antibody) was stained in red and nuclei (DAPI) in blue. (A) Low-magnification view of brain section. Arrow indicates cerebral artery in field of view. (B) Zoomed-in cross section of the somatosensory cortex neurons sections were stained for Panx1 and neurons using NeuN antibody (green). (c) Isolated control PCA sections were collected from Panx1 fl/fl (control) at low magnification, as well as (D) secondary antibody only and (E) rabbit IgC. (F) High magnification of control PCA and PCA from mice that had genotypes of SMC Panx1 $\Delta / \Delta$ (G), and EC Panx1 $\Delta / \Delta(\mathbf{H})$. In D and $\mathbf{E}$, the autofluorescence of the internal elastic lamina is in green and separates the EC (E) and SMC (S) layers. In F-H the separation between EC and SMC, the IEL, is indicated by a dotted line. Asterisks indicate lumen of artery in each image. (A and B) Scale bars: $100 \mu \mathrm{m}$. (C-E) Scale bar: $20 \mu \mathrm{m}$. (F-H) Scale bar: $10 \mu \mathrm{m}$.

Doppler measurements of the MCA during the 90-minute ischemic injury (change in blood flow through the MCA for EC Panx fl/fl mice, $-62.85 \% \pm 7.30 \%$; EC Panx $\Delta / \Delta$ mice, $-61.41 \% \pm 7.66 \%$ ).

Pharmacological inhibition of Panx1 reduces development of cerebral artery myogenic tone. In contrast to venous-circulation ischemic responses, arterial function can also impact recovery following cerebral ischemia/reperfusion injury, particularly by regulating CBF recovery through changes in myogenic tone. Given the dependence of purinergic signaling for myogenic tone development $(18,19)$, we first treated mouse cerebral arteries with apyrase to degrade extracellular purines. Here, we observed that apyrase significantly reduced myogenic tone in C57B1/6 mouse cerebral arteries (Figure 4A) by altering the active diameter (Figure 4B), whereas the passive diameter was not affected by apyrase treatment (Figure 4C). Treatment with carbenoxolone similarly reduced the development of myogenic tone by diminishing the active diameter without changing the passive diameter of cerebral arteries (Figure 4, A-C). Carbenoxolone is a nonspecific inhibitor of Panx1, with other targets including the related connexin-based channels; therefore, we also examined treatment with spironolactone, a recently discovered inhibitor of Panx 1 channels, that does not target connexin- or other Panx isoform-based channels (25). Treatment with spironolactone resulted in a similar reduction of cerebral myogenic tone development (Figure 4, D-F). These data suggest that Panx1 and extracellular purines can regulate cerebral myogenic tone without altering the passive properties of the cerebral vessels.

Genetic deletion of endothelial Panx1 reduces development of cerebral artery myogenic tone. Next, we examined the development of myogenic tone in cerebral arteries of EC Panx $1 \Delta / \Delta$ and SMC Panx $1 \Delta / \Delta$ mice (Figure 5). Deletion of EC Panx1 resulted in decreased myogenic tone development (Figure 5A), similar to that seen in pharmacological experiments, with an increase in active diameter (Figure $5 \mathrm{~B}$ ) and no change in passive diameter (Figure 5C). In addition to the reduced myogenic tone development in the PCA from EC Panx $1 \Delta / \Delta$ mice, a similar reduction is observed in their MCA (Supplemental Figure 1; supplemental material available online with this article; https://doi.org/10.1172/jci.insight.96272DS1). However, deletion of SMC Panx1 did not alter the development of myogenic tone in cerebral arteries (Figure 5, D and E). These data, coupled with the data above, suggest that EC Panx1 may regulate severity of cerebral ischemia/reperfusion injury 


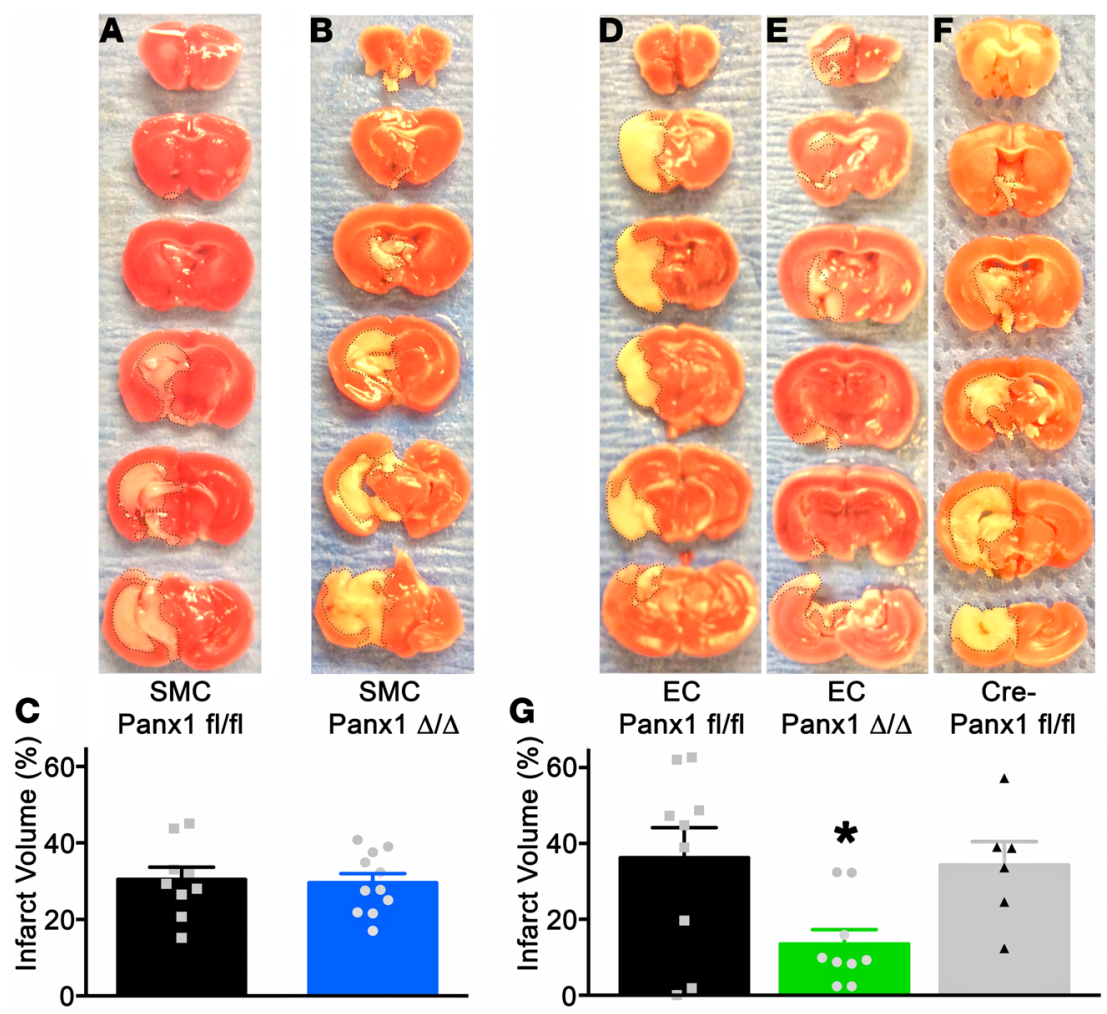

Figure 2. Deletion of EC Panx1 significantly reduces stroke infarct volume. Brains were isolated from mice following a 90-minute MCA occlusion and 24-hour reperfusion and stained with TTC to quantify infarct volume. Representative brains, in 2-mm sections, are shown for SMC Panx fl/fl (32.3\% infarct, A), SMC Panx $\Delta / \Delta$ mice (37.5\% infarct, B), EC Panx $1 \mathrm{fl} / \mathrm{fl}$ (38.9\% infarct, D), EC Panx1 $\Delta / \Delta$ mice (8.7\% infarct, E), and $\mathrm{Cre}^{-} \mathrm{Panx} 1 \mathrm{fl} / \mathrm{fl}$ (33.6\% infarct, F). Quantification of infarct volume is shown for each genotype (C and G). SMC Panx1 fl/fl, $n=9$; SMC Panx1 $\Delta / \Delta, n=11 ;$ EC Panx1 fl/fl, $n=9$; EC Panx1 $\Delta / \Delta$, $n=9$; Cre- Panx1fl/fl, $n=6$ mice. ${ }^{*} P<0.05$; 1 -way ANOVA/Tukey's multiple comparisons post hoc test vs. EC Panx1 fl/fl $(P=0.09$ for EC Panx $1 \Delta / \Delta$ vs. Cre- Panxifl/fl).

through dual mechanisms of increased infiltration of circulating leukocytes into brain tissue through veins and increased myogenic tone in cerebral arteries.

Previous work has demonstrated that mesenteric arteries also utilize purinergic signaling to regulate myogenic tone, and Panx1 is expressed within the ECs of mesenteric arteries $(11,26,27)$. In concordance with those experiments, we found that carbenoxolone treatment, which inhibits both Panx1 and Cx43 channels, significantly reduced myogenic tone development in third-order mesenteric arteries (Figure 6, A-C), whereas spironolactone treatment, which does not inhibit Cx43 channels (25), did not impact mesenteric myogenic tone development (Figure 6, D-F). In agreement with our pharmacological data, deletion of EC Panx1 did not alter development of myogenic tone in mesenteric arteries (Figure 6, G-I). This finding is in line with the previous report that mesenteric myogenic tone development may work through $\mathrm{Cx} 43$ (27). In fact, global Panx1-KO mice show no differences in myogenic tone development in mesenteric arteries, while adrenergic-mediated vasoconstriction is ablated, as described previously (10, 27). To further differentiate mesenteric myogenic tone (which may be important for blood pressure regulation) from cerebral myogenic tone (which is isolated from blood pressure regulation), we used radiotelemetry to measure mean arterial pressure (MAP) and heart rate. Unlike the hypotensive mice lacking Panx1 in SMCs (10), mice lacking EC Panx1 did not show a difference in MAP (Figure 6J) and heart rate (Figure 6K) from control mice.

\section{Discussion}

As tPA administration remains the only approved pharmacological intervention for ischemic stroke in the acute period, a medical need exists for novel therapeutic targets (1). Purinergic signaling has been demonstrated to have important effects on stroke outcomes $(28,29)$. Panx1 channels are potentially novel purine release channels that are expressed on multiple cell types in the brain, including cerebral ECs and SMCs, as we demonstrate here $(5,20,21)$. Previous studies showed that global deletion of Panx1, although leading to compensatory changes of other pannexin isoforms, significantly reduced infarct size following ischemic stroke (6-9). Similar effects were observed using pharmacological pannexin inhibitors, including systemically administered probenecid, a nonspecific inhibitor of pannexins, and a pannexin C-terminal mimetic peptide (6-9). Thus, while pannexins were implicated in improving overall stroke outcomes, these studies lacked cell type-specific targeting of Panx1 and mechanisms contributing to the stroke phenotype. 
A

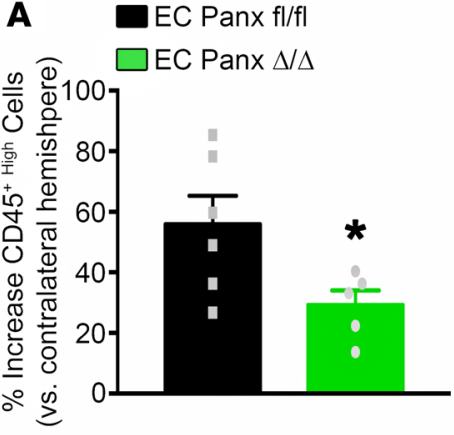

D
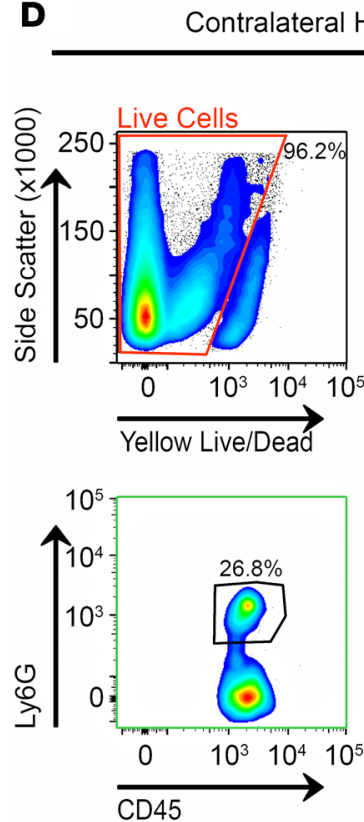

B

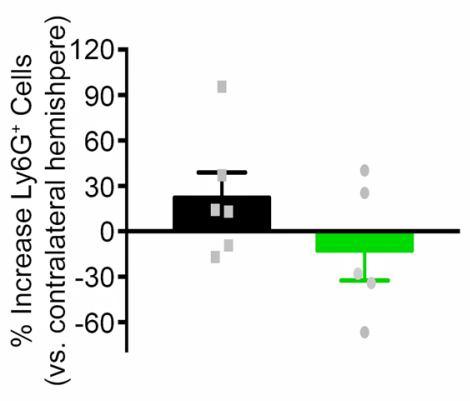

C

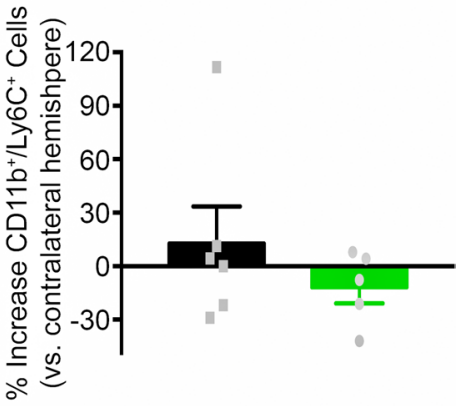

E

Stroked Hemisphere
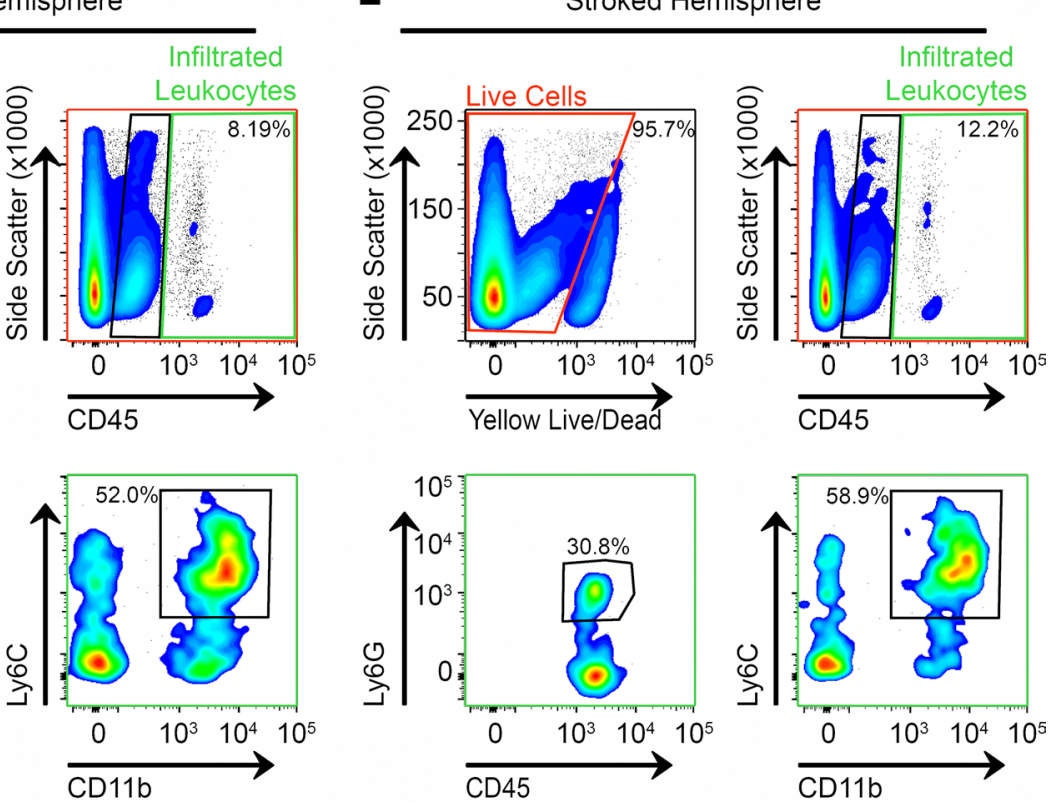

Figure 3. Brain tissue infiltration of leukocytes is significantly reduced in EC Panx1 $\mathbf{1} / \Delta$ mice following stroke. Following a 90 -minute $M C A O$ and

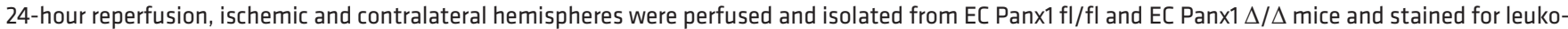
cyte markers for flow cytometry analysis. Differences in the percentages of total infiltrated leukocytes (CD45 hi), neutrophils (CD45 hi $/$ Ly6G+), or monocytes $\left(\mathrm{CD}_{5} 5^{\mathrm{hi}} / \mathrm{CD} 11 \mathrm{~b}^{+} / \mathrm{Ly}_{6 \mathrm{C}}\right)$ between the ischemic and contralateral hemispheres were calculated (A-C). Representative flow cytometry graphs of the contralateral (D) and ischemic (E) hemispheres of an EC Panx $1 \mathrm{fl} / \mathrm{fl}$ mouse are shown, along with gating strategies. Live cells (red box) were further analyzed for expression of CD45. Intermediate expression of CD45 is indicative of microglia cells (black box), while cells expressing high levels of CD45 (green box), indicative of infiltrated leukocytes, were further subdivided into Ly6G-positive or CD11b and Ly6C double-positive cells. EC Panx1 fl/fl, $n=6 \mathrm{mice}$; EC Panx1 $\Delta / \Delta, n=5$ mice. ${ }^{*} P<0.05$; Student's $t$ test.

Therefore, we specifically explored the contributions of SMC and EC Panx1 on the severity of stroke outcome in mice. Here, we demonstrate for the first time to our knowledge that deletion of Panx1 from ECs, but not SMCs, significantly reduces infarct volume following a 90-minute cerebral ischemia and 24-hour reperfusion injury, an effect that is independent of tamoxifen. These effects appear to be mediated through the dual mechanisms of reduced leukocyte infiltration into ischemic brain tissue, as well as reduced development of cerebral arterial myogenic tone.

Endothelial Panx1 and purine release from venous ECs have been implicated in regulation of inflammation following injury. Following TNF- $\alpha$ application, ATP release and subsequent leukocyte adhesion along venous ECs were shown to be Panx1 dependent (11). In addition, TNF- $\alpha$-induced leukocyte adhesion and emigration were significantly reduced in the cremaster vasculature of mice lacking endothelial Panx1 (11). In the context of ischemic stroke, TNF- $\alpha$ secretion is enhanced following stroke onset, while blocking TNF signaling pathways improved stroke outcome $(12,23,24)$. Studies supporting the role of purines in stroke found that deletion of ectonucleotidases, such as CD39 and CD73, resulted in larger infarcts following a cerebral ischemic insult $(28,29)$. The increased infarct volume correlated strongly with an increase 


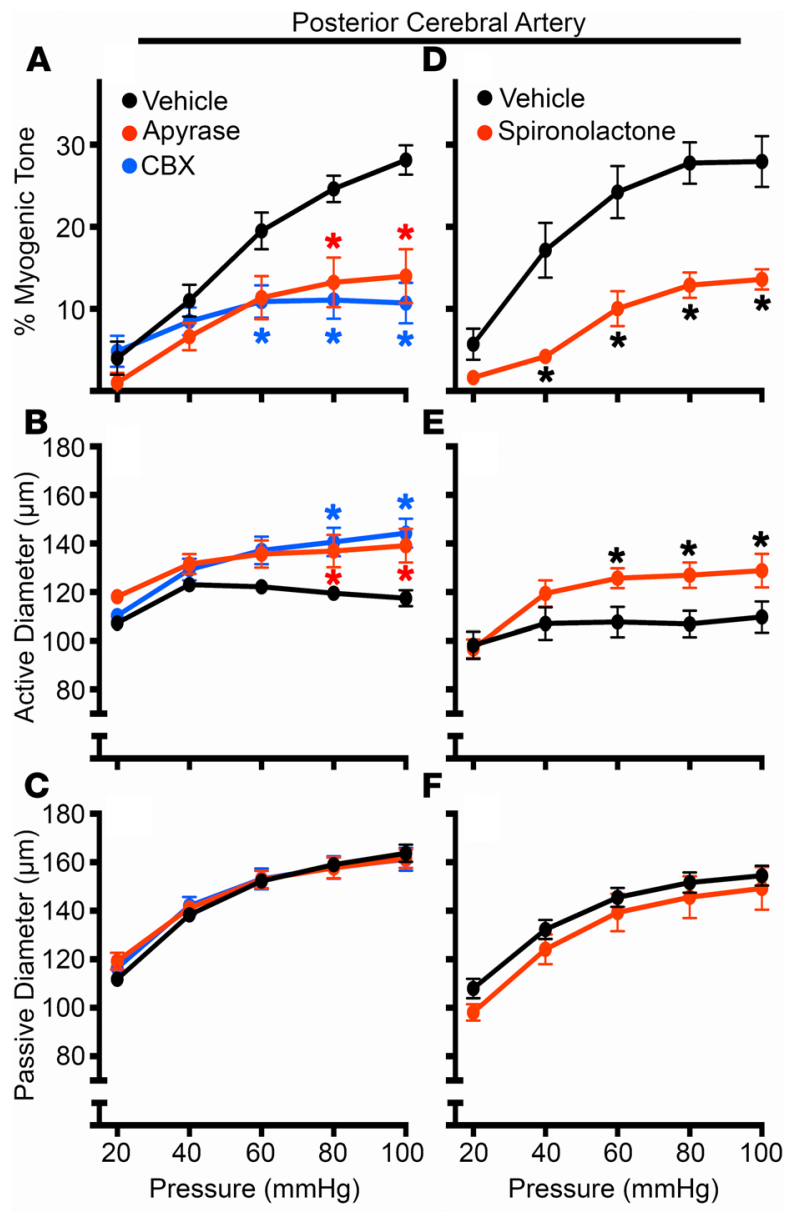

Figure 4. Degradation of extracellular nucleotides or inhibition of Panx1 channels significantly blunts development of cerebral myogenic tone. Isolated PCAs from $C 57 \mathrm{BI} / 6$ control mice were pressurized, and intraluminal pressure was incrementally increased from $20-100 \mathrm{mmHg}$ in the presence or absence of apyrase (10 $\mathrm{U} / \mathrm{ml} ; \mathbf{A}-\mathbf{C})$, carbenoxolone (CBX; $50 \mu \mathrm{M} ; \mathbf{A}-\mathbf{C})$, or spironolactone $(80 \mu \mathrm{M} ; \mathbf{D}-\mathbf{F})$. Percent myogenic tone (A and $\mathbf{D})$, active diameter ( $\mathrm{Ca}^{2+}$ present in solution; $\mathbf{B}$ and $\mathbf{E})$, and passive diameter ( $\mathrm{Ca}^{2+}$-free solution; $\mathbf{C}$ and $\left.\mathbf{F}\right)$ were evaluated over the range of pressures. For each group in $\mathbf{A}-\mathrm{C}, n=6$ mice; Spironolactone, $n=4$; and the vehicle control, $n=7$ mice. ${ }^{*} P<0.05$ vs. vehicle control. Two-way ANOVA/ Sidak's multiple comparisons post hoc test.

in leukocyte infiltration, suggesting the importance of extracellular ATP/ UTP degradation to adenosine in postischemic stroke inflammation (28, 29). More conclusively, our data show that deletion of endothelial Panx1 leads to a significant reduction in leukocyte infiltration into the ischemic hemisphere following 24-hour perfusion, with no significant differences in the relative amounts of the specific subsets of leukocytes, such as neutrophils and monocytes (30). These data suggest that endothelial Panx1 regulates passage of leukocytes from the venous system into the brain tissues in a non-subtype-specific manner following a cerebral ischemia/reperfusion injury; however, there are additional subsets of leukocytes that we have not examined, thus necessitating future evaluation.

Spreading depolarizations (SD) have been found to alter the bloodbrain barrier, resulting in a breakdown of the EC-EC junctions due to increased MMP-9 activity, neuroinflammation, and injury-induced depolarizations, which - combined - can have negative effects on the ischemic and penumbra tissue (31). Recently, neuronal Panx1 has been implicated in regulating SD, where inhibition of the Panx1-P2X7 complex suppressed SD and neuroinflammation (32). Although CBF and neurovascular communication may regulate SD and SD recovery, it is unclear if cerebral endothelial Panx1 regulates SD. Furthermore, although the Panx1-P2X7 complex has been shown to be functionally relevant in neurons, it is unknown if P2X7 functionally complexes with Panx1 in ECs and if this complex is important for the role of the cerebral vasculature during an ischemic stroke injury. Further investigation of the potential roles and binding partners of cerebral endothelial Panx1 is necessary to determine if these neuronal effects are also present in the cerebral ECs.

Our data also demonstrate that EC Panx1 regulates cerebral arterial function. Cerebral arteries are predominately regulated by myogenic reactivity, which maintains constant $\mathrm{CBF}$ rates despite fluctuations in MAP (14). The mechanism of myogenic reactivity in the cerebral circulation remains incompletely understood, but a number of published reports suggest that purinergic receptors play a strong role in regulating myogenic responses(18, 19), specifically the P2Y4 and P2Y6 isoforms (18). As purinergic receptors are activated by extracellular purines such as ATP/UTP and ADP/UDP, this raises the possibility that Panx1 channels might facilitate release of purines needed for receptor activation. In support of this hypothesis, our data show that treatment with apyrase, which degrades extracellular purines, or deletion of EC Panx1 results in a reduction in the myogenic tone development, which was not observed upon deletion of SMC Panx1. Interestingly, this reduction was due to changes in the active but not the passive diameter of cerebral arteries, indicating that the reduction was likely due to cell signaling and not to changes in the vessel structural properties. In line with these observations, inhibition of myogenic tone might also lead to reduced vasoconstriction, increased $\mathrm{CBF}$ within the cerebral vasculature, and improved stroke outcomes, although this remains to be tested. The mechanisms by which Panx1 channels in cerebral arterial ECs are activated also remains to be elucidated. Panx1 channels have been shown to be mechanosensitive, although the data is ambiguous as to whether Panx1 channels open in direct response to stretch themselves or if channel opening is mediated by additional stretch-sensing proteins $(33,34)$.

Myogenic tone development is a common regulatory mechanism in most peripheral resistance arteries, which raises an important question as to whether endothelial Panx1-dependent myogenic reactivity is unique to the cerebral arteries, and if it can be therapeutically targeted without alterations 

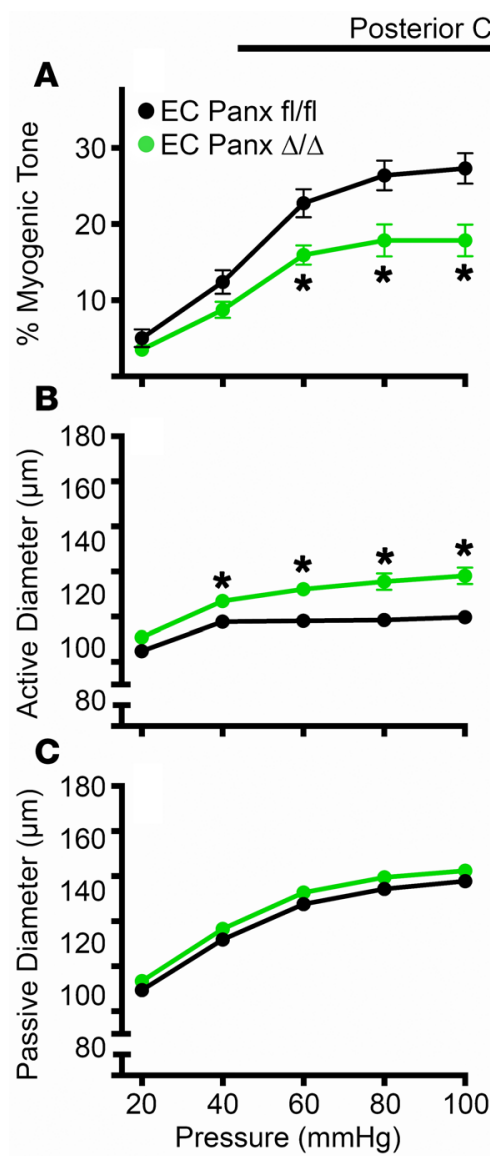
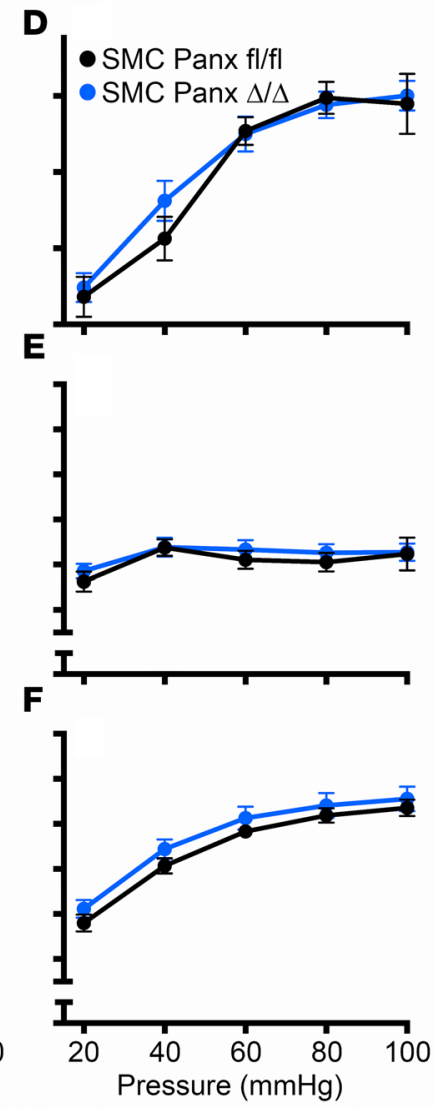

Figure 5. Development of myogenic tone in cerebral arteries is dependent upon EC Panx1 but not SMC Panx1. Percent myogenic tone, active diameter, and passive diameter of isolated, pressurized PCAs from EC Panx1 (fl/fl and $\Delta / \Delta ; \mathbf{A}-\mathbf{C}$ ) and SMC Panx1 (fl/fl and $\Delta / \Delta$; D-F) mice were analyzed. EC Panx1 fl/fl, $n=6$; EC Panx1 $\Delta / \Delta$, $n=8 ;$ SMC Panx $1 \mathrm{fl} / \mathrm{fl}, n=4$; and SMC Panx $1 \Delta / \Delta, n=6$ mice. ${ }^{*} P<$ 0.05 . Two-way ANOVA/Sidak's multiple comparisons post hoc test.

to the peripheral vasculature. Mice treated with carbenoxolone display significantly reduced myogenic tone development in resistance arteries in the cerebral and the mesenteric vasculature, as demonstrated in this work. However, carbenoxolone inhibits connexin 43 ( $\mathrm{Cx} 43)$ channels as well as Panx1 channels (35). Here, we utilized our inhibitor, spironolactone (25), and our transgenic EC Panx1-deleted mice and found that myogenic tone in mesenteric arteries was unaffected by the inhibition of Panx1 (but not Cx43) or the loss of EC Panx1, respectively. These data, along with previously published data, indicate that $\mathrm{Cx} 43$ may participate in myogenic tone development in mesenteric arteries, but more likely, EC Panx1 does not (27). Future studies should elucidate the feasibility of targeting differential myogenic responses between the cerebral vasculature and mesenteric arteries.

Blood pressure is regulated through SMC Panx1, which regulates $\alpha$-adrenergic signaling (10), and mice specifically lacking smooth muscle Panx1 have been found to have a significant reduction in their MAP (10). Changes in overall blood pressure can impact postischemic stroke outcome; how-

ever, the role of EC Panx1 in blood pressure regulation and heart rate was previously unknown. Our data showed that neither MAP during the day when mice are at rest nor at night when mice are active were altered by deletion of endothelial Panx1. Additionally, heart rate was unchanged in mice lacking endothelial Panx1. These data suggest that EC Panx1 regulates cerebral myogenic tone and impacts ischemic stroke outcome independently of changes in peripheral blood pressure.

In summary, we show for the first time to our knowledge that EC Panx1 within the cerebral vasculature regulates pathways important to ischemic stroke outcomes. Leukocyte infiltration was significantly reduced following cerebral ischemic injury when Panx1 was deleted specifically from ECs. In addition, myogenic reactivity in cerebral arteries is regulated by EC Panx1, and deletion of EC Panx1 uniquely reduced myogenic tone development in cerebral arteries with no change in peripheral mesenteric myogenic reactivity or overall blood pressure. Together, these data indicate that endothelial Panx1 influences multiple vascular functions that influence the severity of the stroke phenotype and that differentially targeting EC Panx 1 in the cerebral vasculature may be a novel strategy for improving outcomes following ischemic stroke.

\section{Methods}

Mice. Mice were fed a normal chow and housed under a 12-hour light/dark cycle. All mice were male and were used between 10 and 20 weeks of age. The genotypes include: C57B1/6 purchased from

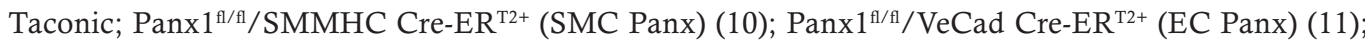
and Panx $1^{\mathrm{A} / \mathrm{fl}} / \mathrm{Cre}^{-}$. Transgenic mice were injected for 10 days with vehicle (peanut oil; [fl/fl]) or tamoxifen $(1 \mathrm{mg} / \mathrm{kg} /$ day $)$ to delete Panx1 from the corresponding cell type $(\Delta / \Delta)$. Panx $1^{\mathrm{A} / \mathrm{fl}} / \mathrm{Cre}^{-}$were injected with tamoxifen to serve as a tamoxifen control $\left(\mathrm{Cre}^{-} \mathrm{Panx} 1 \mathrm{fl} / \mathrm{fl}\right)$.

$I H C$. For whole brain and isolated PCA sections, C57Bl/6 mice were anesthetized using methoxyflurane (Alfa Aesar) and transcardially perfused with heparinized PBS followed by $4 \%$ paraformaldehyde (PFA; MilliporeSigma) in PBS. Whole brains were then placed in 4\% PFA for 24 hours at $4^{\circ} \mathrm{C}$, followed by 48 hours in $30 \%$ sucrose in PBS, embedded in OCT compound, and flash frozen, and $10 \mu \mathrm{m}$ sections were 

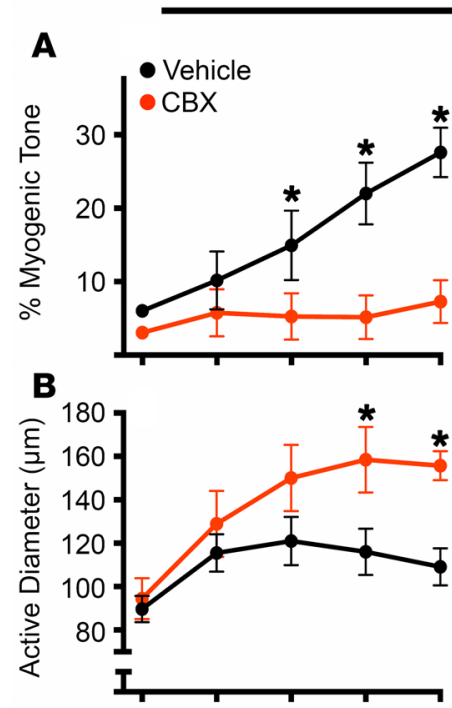

D
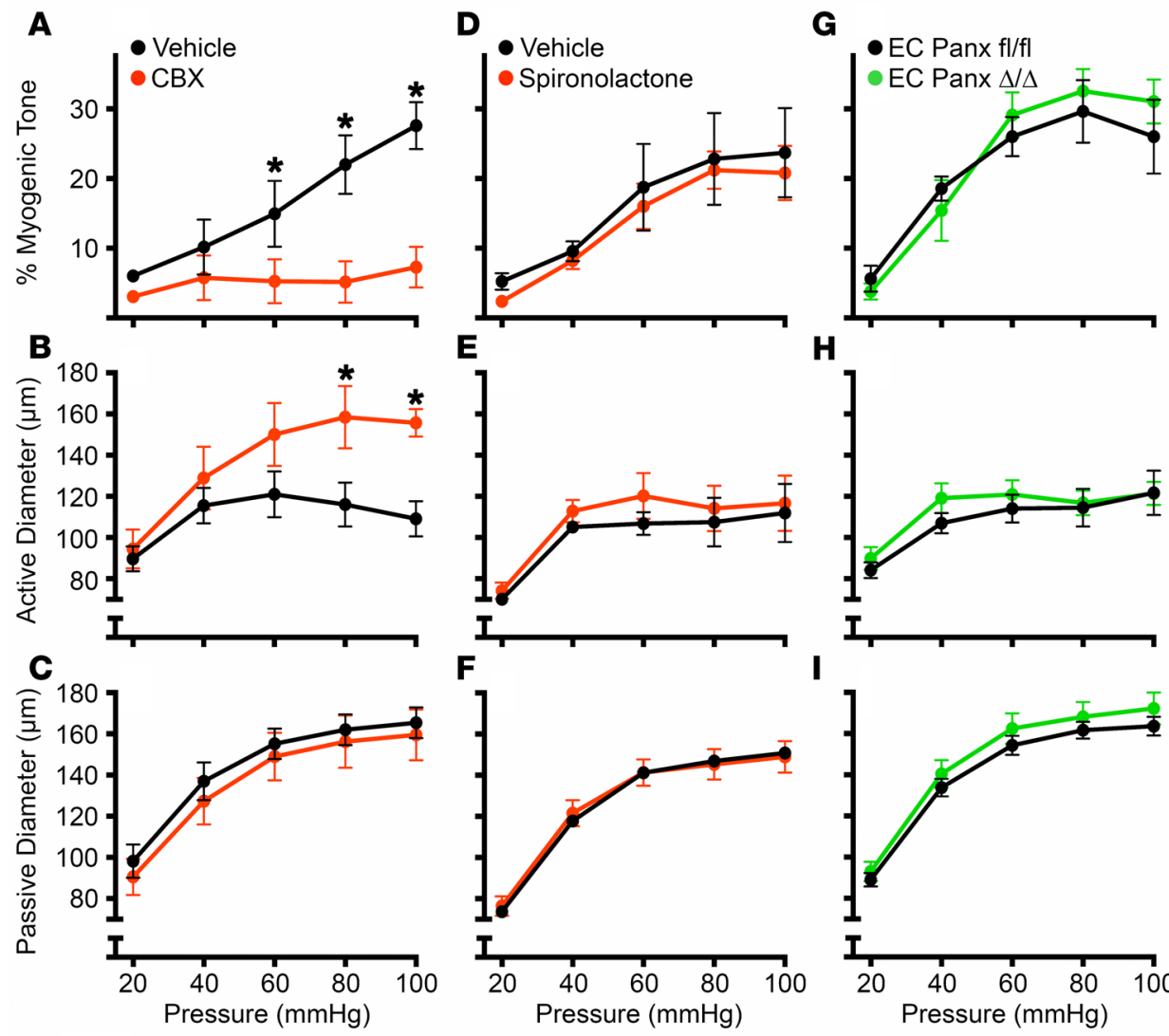

E
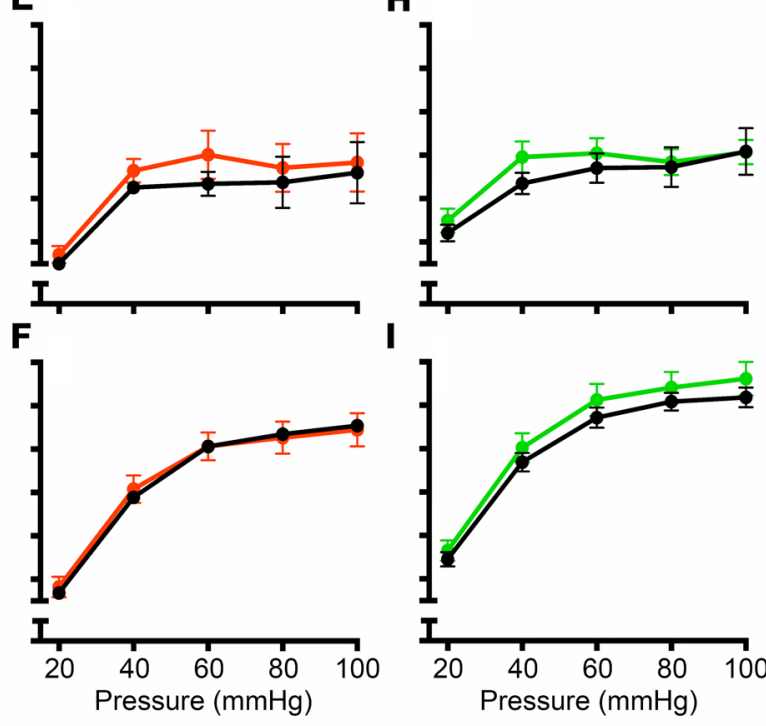

$\mathbf{J}$

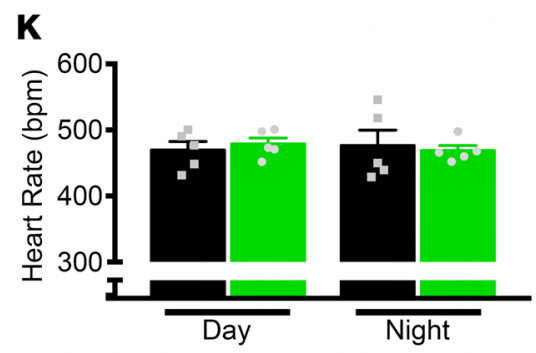

Figure 6. Myogenic tone in mesenteric arteries, mean arterial pressure, and heart rate are independent of EC Panx1. Pressure curves were run on third-order mesenteric arteries isolated from C57BI/6 mice in the presence or absence of carbenoxolone (CBX; $50 \mu \mathrm{M} ; \mathbf{A}-\mathbf{C})$, spironolactone (80 $\mu \mathrm{M}$ D-F), or mesenteric arteries isolated from EC Panx1 (fl/fl and $\Delta / \Delta)$ mice (G-I). Percent myogenic tone, active diameter, and passive diameter were

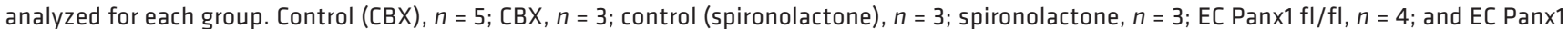
$\Delta / \Delta, n=6$ mice. ${ }^{*} P<0.05$. Two-way ANOVA/Sidak's multiple comparisons post hoc test. Telemetry was used to collect average day and night mean arterial pressure (J) and heart rate (K) from EC Panx1 fl/fl and EC Panx1 $\Delta / \Delta$ mice. No significant differences were found using Student's $t$ test.

obtained. PCAs were isolated from the brain, fixed in 4\% PFA for 1 hour, and embedded in paraffin, and 5 - $\mu \mathrm{m}$ cross sections were obtained. Whole brain sections were brought to room temperature and washed in PBS to remove excess OCT. PCA cross sections were subjected to paraffin removal and washing. Whole brain and PCA cross sections then underwent antigen retrieval (antigen unmasking solution, Vector), were blocked for 30 minutes, and were stained for Panx1 as previously described (4). Whole brain sections were stained with Panx1-CT antibody (1:200) (4), and neuronal-stained brains and isolated PCA sections were stained with Panx1-NT antibody (1:50; Cell Signaling Technology; catalog 91137). Neurons were stained using NeuN (MilliporeSigma; clone MAB377; 1:1,000). Control whole brains were stained with Rabbit IgG control $\mathrm{Ab}(25 \mu \mathrm{g} / \mathrm{ml}$; catalog I-1000, Vector Laboratories). Anti-rabbit secondary F(ab)'2 antibody coupled to Alexa Fluor 568 (1:400; Invitrogen) was applied to all sections (except neuronal stained sections: 
Alexa Fluor 488 goat anti-mouse [NeuN] and Alexa Fluor 555 goal anti-rabbit [Panx1]) and then imaged using an Olympus FluoView 1000 as previously described (36).

$M C A O /$ reperfusion. Three weeks following the last injection of tamoxifen, to ensure removal of tamoxifen from the system, mice underwent a 90-minute MCAO followed by a 24-hour reperfusion as previously described (37). Briefly, under anesthesia, a 6-0 filament was fed through the left external carotid artery and placed over the MCA to occlude the MCA for 90 minutes. Anesthesia was stopped once the MCA was occluded, and then mice were reanesthetized after 90 minutes to remove the suture. Twenty-four hours after removal of the suture, the brain was harvested, and 2-mm sections were stained with TTC to visualize the ischemic region vs. the remaining healthy tissue. Using ImageJ, infarct volume (corrected for edema) was calculated as: Percent Infarct volume $=([$ nonischemic hemisphere area $-($ ischemic hemisphere area infarct area) $\times 100$ ) / nonischemic hemisphere area.

Flow cytometry. Flow cytometry was used to determine leukocyte infiltration into the brain (30). Mice were anesthetized using isoflurane and were transcardially perfused with $10 \mathrm{ml}$ heparinized PBS, separated into ischemic vs. nonischemic hemispheres, and then placed into HBSS containing divalent cations $(\mathrm{HBSS}+\mathrm{Ca} / \mathrm{Mg}$ ). To obtain a single cell suspension, hemispheres were pushed through a $100-\mu \mathrm{m}$ filter using the plunger of a 5-ml syringe, and the filter was rinsed continuously with $\mathrm{HBSS}+\mathrm{Ca} / \mathrm{Mg}$. The samples were then centrifuged at $300 \mathrm{~g}$ at $4^{\circ} \mathrm{C}$ for 5 minutes, and the pellet was resuspended in $\mathrm{HBSS}+$ $\mathrm{Ca} / \mathrm{Mg}$ containing liberase TL ( $2 \mathrm{U} / \mathrm{ml}$; MilliporeSigma). Samples were digested for 1 hour at $37^{\circ} \mathrm{C}$ with continuous shaking at $300 \mathrm{rpm}$ and then filtered through a $70-\mu \mathrm{m}$ filter. The filter was then rinsed with 3 $\mathrm{ml}$ HBSS containing DNase1 (666 U/ml; MilliporeSigma) and FBS (10\%), followed by $15 \mathrm{ml}$ of wash buffer (HBSS containing 10\% FBS). Following centrifugation at $300 \mathrm{~g}$ at $18^{\circ} \mathrm{C}$ for 5 minutes, samples were resuspended in room-temperature, $25 \%$ density gradient medium (DGM) (for 100\% DGM: mix 1 part $1.5 \mathrm{M} \mathrm{NaCl}$ and 9 parts Percoll [MilliporeSigma]; diluted to $25 \%$ by addition of HBSS containing $3 \%$ FBS). The samples were then centrifuged at $600 \mathrm{~g}$ at $10^{\circ} \mathrm{C}$ for 20 minutes using the lowest acceleration rate of the Sorvall ST16R centrifuge (with Thermo Fisher Scientific rotor 75003181) and no brake. The layer of myelin was carefully aspirated, and the remaining pellet was rinsed in washing buffer, moved to a clean tube, and centrifuged at $300 \mathrm{~g}$ at $10^{\circ} \mathrm{C}$ for 5 minutes. Cell pellet was resuspended in Yellow live/ dead cell stain (Thermo Fisher Scientific) per manufactures directions, incubated at $4^{\circ} \mathrm{C}$ for 15 minutes, and then centrifuged at $300 \mathrm{~g}$ at $10^{\circ} \mathrm{C}$ for 5 minutes. The cell pellet was then resuspended in wash buffer and blocked using anti-murine CD16/32 FC receptor $\left(2.5 \mu \mathrm{g} / \mathrm{ml}\right.$; Invitrogen) for 10 minutes at $4^{\circ} \mathrm{C}$. Fluorophore-conjugated primary antibodies were then added to the cells and incubated for 20 minutes at $4^{\circ} \mathrm{C}$ in the dark. The following antibodies were used; FITC anti-mouse CD45 Ab (clone 30-F11; 5 $\mu \mathrm{g} / \mathrm{ml}$; BioLegend), APC/Cy7 anti-mouse Ly6G Ab (clone 1A8; $1 \mu \mathrm{g} / \mathrm{ml}$; BioLegend), Brilliant Violet 421 anti-mouse/human CD11b Ab (clone M1/70; $2 \mu \mathrm{g} / \mathrm{ml}$; BioLegend), and PE anti-mouse Ly6C Ab (clone HK1.4; $1 \mu \mathrm{g} / \mathrm{ml}$; BioLegend). Cells were then rinsed in FACS Buffer (PBS containing 3\% FBS), centrifuged at $400 \mathrm{~g}$ at $4^{\circ} \mathrm{C}$ for 7 minutes, and resuspended in FACS buffer. All samples were then analyzed with the Cytek FACSCalibur benchtop analyzer, and data were analyzed using FlowJo v10. Gates were determined using single-stained UltraComp eBeads (eBioscience), per manufactures directions, and fluorescence minus one (FMO) control samples, which contained all fluorescent probes except one.

Pressure myography. Freshly isolated PCAs and superior cerebellar arteries (SCAs), MCAs, or third order mesenteric arteries were placed into ice-cold Krebs-HEPES solution containing (in $\mathrm{ml}$ ) $\mathrm{NaCl}$ 118.4, $\mathrm{KCl} 4.7, \mathrm{MgSO}_{4} 1.2, \mathrm{NaHCO}_{3} 4, \mathrm{KH}_{2} \mathrm{PO}_{4} 1.2, \mathrm{CaCl}_{2} 2$, Hepes 10, and glucose 6. The vessels were then mounted in a pressure arteriograph (Danish MyoTechnology) with the lumen filled with Krebs-HEPES solution. The vessels were pressurized to $80 \mathrm{mmHg}$ and heated to $37^{\circ} \mathrm{C}$ for 30 minutes. For experiments treated with pharmacological inhibitors, lumen and bath solutions were changed to contain the desired concentration of drug or control diluent and allowed to equilibrate for 30 minutes. Myogenic curves were completed by increasing the luminal pressure from $20-120 \mathrm{mmHg}$ in $20-\mathrm{mmHg}$ increments to obtain the active diameter of the vessel. The pressure steps were then repeated after washing with a $\mathrm{Ca}^{2+}$-free Krebs-HEPES solution supplemented with $1 \mathrm{mM}$ EGTA and $10 \mu \mathrm{M}$ sodium nitroprusside to obtain maximal passive diameters for each pressure. Myogenic tone values are calculated as: ([passive diameter - active diameter] / passive diameter) $\times 100$.

Radiotelemetry. Ten- to 12-week-old mice were implanted with radiotelemetry units (Data Sciences International [DSI]) as previously described $(10,38)$. Briefly, while under isoflurane anesthesia, the catheter of the radiotelemetry unit was placed in the left carotid artery and advanced toward the heart until resistance was felt. 
The radiotransmitter was then placed in a s.c. pouch on the right flank. Mice were allowed to recover for 5 days. Blood pressure was then continuously recorded for 7 days.

Statistics. Data were analyzed using Prism 7 software (GraphPad Software Inc.) and displayed in mean \pm SEM. Two-tailed Student's $t$ tests or 1-way ANOVA followed by Tukey's multiple comparisons post hoc tests were used where appropriate. Two-way ANOVA followed by Sidak's multiple comparisons post hoc tests were used for pressure myography experiments. $P<0.05$ was considered to be statistically significant.

Study approval. All mice and procedures were approved by the University of Virginia School of Medicine ACUC.

\section{Author contributions}

MEG designed the research project, conducted experiments, analyzed and interpreted the results, and wrote and approved the manuscript. SAE designed the research project and conducted myogenic tone experiments. JL performed experiments associated with stroke procedures. HMB conducted flow cytometry experiments and analyzed blood pressure data. SML, JTB, and TJJ performed myogenic tone experiments. RPG and MKP performed IHC studies. ZZ and BEI designed the project and wrote and approved the manuscript. All authors read and provided comments on the manuscript.

\section{Acknowledgments}

The authors thank the members of the Pannexin Interest Group at the University of Virginia for their aid and input throughout the development of this work, as well as Alexander S. Keller, Leon J. DeLalio, and T.C. Stevenson Keller IV for critical feedback. The authors also thank the Flow Cytometry Facility at the University of Virginia School of Medicine for their assistance with flow cytometry experiments and the University of Virginia Histology core for processing vessels and brains for sectioning and mounting. This work was supported by NIH training grant HL007284 (MEG), NIH F32 HL131399 (MEG), NIH P01 HL120840 (BEI), the Robert M. Epstein Professorship endowment, University of Virginia (ZZ), and NCI P30 CA044579-23 (Flow Cytometry Center grant).

Address correspondence to: Brant E. Isakson, P.O. Box 801394, Charlottesville, Virginia 22908, USA. Phone: 434.924.2093; Email: brant@virginia.edu.

1. Go AS, et al. Heart disease and stroke statistics--2014 update: a report from the American Heart Association. Circulation 2014;129(3):e28-e292.

2. Fugate JE, Rabinstein AA. Update on intravenous recombinant tissue plasminogen activator for acute ischemic stroke. Mayo Clin Proc. 2014;89(7):960-972.

3. Bond SR, Naus CC. The pannexins: past and present. Front Physiol. 2014;5:58.

4. Lohman AW, et al. Expression of pannexin isoforms in the systemic murine arterial network. J Vasc Res. 2012;49(5):405-416.

5. Burns AR, Phillips SC, Sokoya EM. Pannexin protein expression in the rat middle cerebral artery. J Vasc Res. 2012;49(2):101-110

6. Xiong XX, et al. Probenecid protects against transient focal cerebral ischemic injury by inhibiting HMGB1 release and attenuating AQP4 expression in mice. Neurochem Res. 2014;39(1):216-224.

7. Bargiotas P, et al. Pannexins in ischemia-induced neurodegeneration. Proc Natl Acad Sci USA. 2011;108(51):20772-20777.

8. Weilinger NL, et al. Metabotropic NMDA receptor signaling couples Src family kinases to pannexin-1 during excitotoxicity. Nat Neurosci. 2016;19(3):432-442.

9. Lohman AW, Isakson BE. Differentiating connexin hemichannels and pannexin channels in cellular ATP release. FEBS Lett. 2014;588(8):1379-1388.

10. Billaud M, et al. A molecular signature in the pannexin1 intracellular loop confers channel activation by the $\alpha 1$ adrenoreceptor in smooth muscle cells. Sci Signal. 2015;8(364):ra17.

11. Lohman AW, et al. Pannexin 1 channels regulate leukocyte emigration through the venous endothelium during acute inflammation. Nat Commun. 2015;6:7965.

12. Yang GY, Gong C, Qin Z, Ye W, Mao Y, Bertz AL. Inhibition of TNFalpha attenuates infarct volume and ICAM-1 expression in ischemic mouse brain. Neuroreport. 1998;9(9):2131-2134.

13. Härtl R, Schürer L, Schmid-Schönbein GW, del Zoppo GJ. Experimental antileukocyte interventions in cerebral ischemia. J Cereb Blood Flow Metab. 1996;16(6):1108-1119.

14. Koller A, Toth P. Contribution of flow-dependent vasomotor mechanisms to the autoregulation of cerebral blood flow. $J$ Vasc Res. 2012;49(5):375-389.

15. Lin MP, Sanossian N. Reperfusion therapy in the acute management of ischemic stroke. Cardiol Clin. 2015;33(1):99-109.

16. Lum C, et al. Computed tomographic angiography and cerebral blood volume can predict final infarct volume and outcome after recanalization. Stroke. 2014;45(9):2683-2688.

17. Cipolla MJ, Chan SL, Sweet J, Tavares MJ, Gokina N, Brayden JE. Postischemic reperfusion causes smooth muscle calcium sensitization and vasoconstriction of parenchymal arterioles. Stroke. 2014;45(8):2425-2430. 
18. Brayden JE, Li Y, Tavares MJ. Purinergic receptors regulate myogenic tone in cerebral parenchymal arterioles. J Cereb Blood Flow Metab. 2013;33(2):293-299.

19. Mederos y Schnitzler M, et al. Gq-coupled receptors as mechanosensors mediating myogenic vasoconstriction. EMBO J. 2008;27(23):3092-3103.

20. Vogt A, Hormuzdi SG, Monyer H. Pannexin1 and Pannexin2 expression in the developing and mature rat brain. Brain Res Mol Brain Res. 2005;141(1):113-120.

21. Bruzzone R, Hormuzdi SG, Barbe MT, Herb A, Monyer H. Pannexins, a family of gap junction proteins expressed in brain. Proc Natl Acad Sci USA. 2003;100(23):13644-13649.

22. Yilmaz G, Granger DN. Leukocyte recruitment and ischemic brain injury. Neuromolecular Med. 2010;12(2):193-204

23. Wang Q, Tang XN, Yenari MA. The inflammatory response in stroke. J Neuroimmunol. 2007;184(1-2):53-68.

24. Kim JY, Kawabori M, Yenari MA. Innate inflammatory responses in stroke: mechanisms and potential therapeutic targets. Curr Med Chem. 2014;21(18):2076-2097.

25. Good ME, et al. Pannexin 1 Channels as an Unexpected New Target of the Anti-Hypertensive Drug Spironolactone. Circ Res. 2018;122(4):606-615.

26. Koltsova SV, et al. Myogenic tone in mouse mesenteric arteries: evidence for $\mathrm{P} 2 \mathrm{Y}$ receptor-mediated, $\mathrm{Na}(+), \mathrm{K}(+), 2 \mathrm{Cl}(-)$ cotransport-dependent signaling. Purinergic Signal. 2009;5(3):343-349.

27. Kauffenstein G, et al. Central Role of P2Y6 UDP Receptor in Arteriolar Myogenic Tone. Arterioscler Thromb Vasc Biol. 2016;36(8):1598-1606

28. Petrovic-Djergovic D, et al. Tissue-resident ecto-5' nucleotidase (CD73) regulates leukocyte trafficking in the ischemic brain. $J$ Immunol. 2012;188(5):2387-2398.

29. Pinsky DJ, et al. Elucidation of the thromboregulatory role of CD39/ectoapyrase in the ischemic brain. J Clin Invest. 2002;109(8):1031-1040

30. Posel C, Moller K, Boltze J, Wagner DC, Weise G. Isolation and Flow Cytometric Analysis of Immune Cells from the Ischemic Mouse Brain. J Vis Exp. 2016;(108):53658.

31. Ayata C, Lauritzen M. Spreading Depression, Spreading Depolarizations, and the Cerebral Vasculature. Physiol Rev. 2015;95(3):953-993

32. Chen SP, et al. Inhibition of the P2X7-PANX1 complex suppresses spreading depolarization and neuroinflammation. Brain. 2017;140(6):1643-1656.

33. Bao L, Locovei S, Dahl G. Pannexin membrane channels are mechanosensitive conduits for ATP. FEBS Lett. 2004;572(1-3):65-68.

34. Beckel JM, et al. Mechanosensitive release of adenosine 5'-triphosphate through pannexin channels and mechanosensitive upregulation of pannexin channels in optic nerve head astrocytes: a mechanism for purinergic involvement in chronic strain Glia. 2014;62(9):1486-1501.

35. Good ME, Begandt D, DeLalio LJ, Keller AS, Billaud M, Isakson BE. Emerging concepts regarding pannexin 1 in the vasculature. Biochem Soc Trans. 2015;43(3):495-501.

36. Billaud M, Lohman AW, Straub AC, Parpaite T, Johnstone SR, Isakson BE. Characterization of the thoracodorsal artery: morphology and reactivity. Microcirculation. 2012;19(4):360-372

37. Li L, Zuo Z. Glutamate transporter type 3 knockout reduces brain tolerance to focal brain ischemia in mice. J Cereb Blood Flow Metab. 2011;31(5):1283-1292.

38. Keller TC, et al. Modulating Vascular Hemodynamics With an Alpha Globin Mimetic Peptide (HbaX). Hypertension. 2016;68(6):1494-1503. 